\title{
SURVEY OF SOME PLANTS IN NAINAMALAI FOOTHILL, EASTERN GHATS, NAMAKKAL
}

\author{
SHARMILA M, RAJESWARI M, INDHIRAMUTHU JAYASHREE, TAMIZHARASI K
}

Department of Botany, Vellalar College for Women, Thindal, Erode, Tamil Nadu, India. Email: sharmiselva88@gmail.com

Received: 03 February 2018, Revised and Accepted: 07 May 2018

\begin{abstract}
Objectives: This study was designed to document some medicinal plants in Nainamalai foothill.

Methods: The plants were collected from different sites of the hill area, identified by their local names with the help of villagers while walking through the vegetation. The photographs of these plant species were taken during the field visits.

Results: Plant survey at Ninamalai foothill has documented a total of 124 plants. Among 124 species surveyed, 123 were angiospermic plants and one pteridophytic plant. Analysis of plants showed that the more number of dicotyledons (115 species) belongs to 43 families and 8 monocotyledons are represented in 5 families. Within dicotyledons, 53 species are polypetalae, 34 species are gamopetalae, and 28 monochlamydeae. For each species, botanical name, family, habit, local name, parts used, and medicinal usage are identified and presented. The family-wise analysis showed that the family Amaranthaceae held the dominant position by representing more number of species (13 species). Habit-wise analysis shows that maximum representatives to the plants are from herb (76) community, followed by trees (22), shrubs (17), climbers (6), parasitic shrub and parasitic twinner (1), and fern (1). The absence of epiphytes indicates the loss special habitats due to the degraded status of the hill. Part-wise analysis showed the predominant use of whole plants, followed by leaves, root, seed, bark, fruit, flower, and stem.
\end{abstract}

Conclusion: People of the study area mostly administered phytomedicine orally. A majority of remedies are prepared in the form of extract or juice, followed by powder form, decoction, and freshly collected plant parts. Most of the villagers in the study are poor, and they largely depend on plants for food, medicine, fuel, and other daily necessities.

Keywords: Nainamalai, Dicotyledons, Amaranthaceae, Tree, Epiphytes, Leaves.

(C) 2018 The Authors. Published by Innovare Academic Sciences Pvt Ltd. This is an open access article under the CC BY license (http://creativecommons. org/licenses/by/4. 0/) DOI: http://dx.doi.org/10.22159/ajpcr.2018.v11i10.25097

\section{INTRODUCTION}

Human evolution in growth has advanced due to the outcome of total interaction with the environment, including plants and animals. Prehistoric man documented the great diversity of plants accessible to him. Living intimate to nature, traditional societies have attained inimitable experience about the use of wild flora and fauna. Most of the species are unknown to the people because they live away from such a natural forest ecosystem. Later years of observations and exploration, trials, error, experimentation, or even use of natural methods, the novel member of human communities has recognized whether they are useful or toxic members. Plants are the mainstay of life on earth and a vital resource for human welfare. The close association between the human and plants has evolved over generations of experience. This trend has been continued to the present time where people get much of their needs, particularly, the food and medicine from biological resources [1].

For millennia, the herbs, animals, rocks, and trees were the only pharmaceutical giants we had. For thousands of years, mankind is using plant source to alleviate or cure illnesses. Like all living things on earth, every one of us is still a shareholder in nature - the greatest pharmacy on the earth. The plants represent an enormous pool of natural resources that can produce various products and chemicals for the advantage of all other life forms. Plants are the most formidable chemists. They are constantly producing an arsenal of chemical compounds, to respond to different challenges and threats in their environment [2].

India is one of the most medico-culturally diverse countries in the world. India has a rich diversity of medicinal plants. During the past two decades, some substantial progress has been made in the field of medicinal plants and their traditional use in different parts of India [3]. The Indian subcontinent is a vast repository of medicinal plants that are used in traditional medical treatments; around 20,000 medicinal plants have been recorded. The Indian systems of medicine use around 8000 species of plants which include trees (33\%), herbs $(32 \%)$, shrubs (20\%), climbers (12\%), and epiphytes, grasses, lichens, ferns, and algae put together (3\%) [4]. It is also believed that the people in ancient time were healthier than that of today that is only because of their lifestyle and harmony with the nature. The villagers have their own remedies for medicinal treatment using various plants or plant products present in their near neighborhood area. The traditional use of plants as medicines is well known among the native groups of the area because most of the population of India lives in villages and is economically poor. Due to the lack of modern medical facilities, they use plants to get rid of different ailments.

Crude drugs are usually the dried parts of medicinal plants that form the essential raw materials for the production of traditional remedies of Ayurveda, Siddha, Unani, Homeopathy, Tibetan, and other systems of medicine including the folk, ethno, or tribal medicines. Considering a sharp shrinkage of biological species all around the globe and the increasing commercial values of medicinal plants are confirming the ethnobotanical knowledge and use of plants to treat various ailments and to preserve these natural resources.

India is tenth among the plant-rich countries of the world, fourth among the Asian countries. Moreover, India is also one among the 12 mega biodiversity centers of the world by having 47,000 plants species. The Eastern Ghats is one such a place. The average elevation of the Eastern Ghats is about $750 \mathrm{~m}$, though individual peaks rise to heights of $1672 \mathrm{~m}$ [5]. Toward the south, the Eastern Ghats run in a westward direction meeting the Western Ghats in the Nilgiris of Tamil Nadu. The vegetation of Eastern Ghats is remarkable, and as a whole, the vegetation is typically deciduous type and scrub jungle in most places. Nainamalai foothills are situated on the Eastern Ghats at an altitude of $1200 \mathrm{~m}$ in the Namakkal district 
and are $45 \mathrm{~km}$ from Namakkal town (Plate 1). In the present study, an attempt has been made to enumerate the diversity of medicinal plants in Nainamalai, which is located in Thirumalaipatti village, a plateau of Eastern Ghats, situated in the northern part of Namakkal district, Tamil Nadu, India. Nainamalai Varadharaja Swamy is one of the most ancient temples in Tamil Nadu. This temple is called as "Thirupathi of Salem." Main deities are Sri Varadharaja Swamy and Kuvalayavali Thayar. The temple is situated at a hilltop. The hilltop can be reached by climbing 3500 steps which would hardly take $3 \mathrm{~h}$ trek up the hill.

Complete information of survey, documentation, and inventory of wild medicinal plants in the Nainamalai foothills of Eastern Ghats is meager. In view of this present study, an attempt was made to survey and document the wild medicinal plant species in the foothill area. Therapeutic values and medicinal plants used are also documented.

\section{METHODS}

\section{Survey of Nainamalai foothill, Eastern Ghats, Tamil Nadu, India} Description of the study area

The present study was conducted a survey in the Nainamalai, a plateau of Eastern Ghats, situated in the northern part of Namakkal district, Tamil Nadu, India. Nainamalai foothill is known for medicinal herbs and plants that grow in abundance on the hill slopes. Field trips were conducted from 2015 to 2016. Field trips were made in different seasons to collect medicinal plants.

\section{Field observation}

The plants were collected from different sites of the hill area, identified by their local names with the help of villagers while walking through the vegetation. The photographs of these plant species were taken during the field visits. During the period of the study, regular surveys were undertaken to document the list of wild medicinal plants and their traditional uses. The medicinal plants were also collected during the field survey, identified, and photographed.

The collected plant materials were named in a field book, and number and the field characters such as habit, habitat, color, and odor of flowers, period of flowering and fruiting, occurrence, and other relevant ecological features were observed and are noted in the field book. Proper data regarding each plant species were also collected by assigning botanical name, family, local names, habit, useful parts and medicinal uses. The collected data were entered into an Excel spreadsheet and summarized. Descriptive statistical methods such as percentage were employed, and graphs and tables show the results generated. The plants collected and tagged from foothills around the year were dried and duplicated. Representative samples of medicinal species collected from the study area were preserved as herbarium as per standard methods [6,7]. Each specimen was carefully examined in fresh condition and checked. The taxonomic identification of the plant specimens was done with the help of local and regional floras $[8,9]$. The collected plant specimens were deposited in the Department of Botany, Vellalar College for Women (Autonomous), Erode, Tamil Nadu, for future reference.

\section{RESULT}

In India, the use of plants as medicine dates back to 5000 years. Since times immemorial, medicinal plant nature's veiled and, to a large extent, unexplored nature's pharmacy have been used well-nigh in all human cultures around the world as a basis of safe and effective medicine. Phytochemical and pharmacological feedbacks can be headed to the evidence of possible therapeutic use of medicinal plants and the expansion of novel medicines. No survey of naturally growing medicinal plants of the present study area of Nainamalai, Eastern Ghats, Tamil Nadu, has been reported till date.

\section{Survey of Nainamalai Eastern Ghats}

In the present study, an attempt has been made to catalog the diversity of medicinal plants in the foothill of Nainamalai, Namakkal district. The study clearly indicates that the foothill of Nainamalai has a great diversity

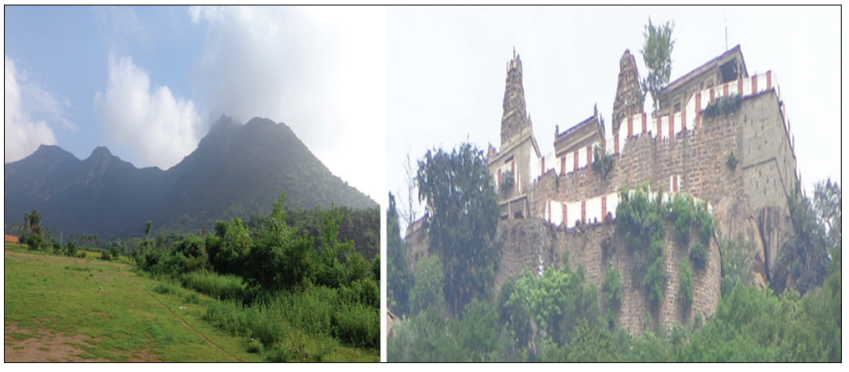

Plate 1: View of Nainamalai (Hill)

of medicinal plants with rich properties which make well of varied kinds of ailments. Most of the medicinal plants hold therapeutic properties in parts such as leaves, roots, stem, fruit, bark, seeds, and flowers.

The study was carried out during 2015-2016, and 124 plant species were collected and identified. The species are arranged in alphabetical order. For each species, botanical name, family, habit, local name, parts used, and medicinal usage are identified and presented (Table 1). The botanical investigation has also led to the documentation of a large number of wild plants used by local villagers.

\section{Analysis of plant diversity and enumeration}

The entire survey revealed that a total of 124 medicinal plant species, distributed among 100 genera belonging to 49 families, were recorded. Among the 124 plants surveyed in the foothill of Nainamalai, 115 dicotyledons, 8 monocotyledons, and one species belonging to pteridophytes were noted, and their percentage is also given (Fig. 1).

Among 115 species from 43 families of dicotyledons, 53 species from polypetalae, 34 from gamopetalae, and 28 from monochlamydeae were also noted. 8 monocotyledons were also represented from 5 families. The family-wise analysis showed that the family Amaranthaceae held the dominant position by representing more number of species (13 species), Euphorbiaceae with 9 species, Fabaceae with 7 species, Acanthaceae and Aizoaceae 6 species each, Malvaceae with 5 species, Mimosaceae and Verbenaceae 4 species each, Asclepiadaceae, Capparidaceae, Lamiaceae, Solanaceae, Apocynaceae, Convolvulaceae, Moraceae, and Liliaceae 3 species each, Asteraceae, Rhamnaceae, Tiliaceae, Meliaceae, Menispermaceae, Pedaliaceae, Sapindaceae, Vitaceae, Cucurbitaceae, Papilionaceae, Portulacaceae, Nyctaginaceae, and Cyperaceae with 2 species each, and the rest of the families are represented with single number of species.

\section{Growth form and plant parts used}

Habit-wise analysis indicates that herbs held the dominant position which was followed by trees, shrubs, climbers, and ferns. Parasitic shrub and parasitic twiner were also present. Among the recorded species, $61 \%$ herbs (76) were found to be dominating over $18 \%$ trees (22), $13 \%$ shrubs (17), $9 \%$ climbers (6), and $1 \%$ fern (1), followed by parasitic $1 \%$ shrub (1) and 1\% parasitic twiner (1) (Fig. 2). In the study area, the highest number from 93 species (55\%) of plants were whole plants used for the preparation of medicine, followed by leaves from 27 species (16\%), roots from 16 species (10\%), seed from 14 species $(80 \%)$, bark from 8 species (4\%), fruits from 6 species (4\%), flower from 2 species (2\%), and stem from 1 species (1\%) (Fig. 2).

A total of 124 plant species distributed in 49 families during the field survey were found to be used as traditional medicine for various disorders and diseases such as anti-inflammatory, anthelmintic, analgesic, leprosy, gonorrhea, bronchitis, diarrhea, dysentery, leukoderma, diabetes, skin diseases, antimutagenic, anticancer, smallpox, seminal weakness, antiplasmodia, asthma, pneumonia, rheumatism, ulcer, malaria, snakebite, piles, rabies, influenza, eye diseases, kidney disorder, wound, jaundice, hepatitis, blood pressure, leprosy, dysentery, dyspepsia, anti-anaphylactic, antispasmodic, nervous disorder, throat infection, anti-asthma, intestinal astringent, rheumatic joint, dental treatment, urinary infection, intestinal 
Table: 1 Survey of medicinal plants in Nainamalai

\begin{tabular}{|c|c|c|c|c|c|}
\hline Botanical name & Family name & Local name & Habit & Parts used & Medicinal uses \\
\hline Abrus precatorius L. & Fabaceae & Kundumani and gunj & Climber & Leaf seed & $\begin{array}{l}\text { Fever, cold, cough, eye } \\
\text { diseases }\end{array}$ \\
\hline Abutilon indicum G. Don. & Malvaceae & Thuththi & $\begin{array}{l}\text { Woody } \\
\text { Herb }\end{array}$ & Whole plant & $\begin{array}{l}\text { Anti-inflammatory, } \\
\text { anthelmintic, analgesic, } \\
\text { leprosy, gonorrhea }\end{array}$ \\
\hline Acacia arabica Willd. & Mimosaceae & Karuvelam & Tree & Whole plant & $\begin{array}{l}\text { Bronchitis, diarrhea, } \\
\text { dysentery, bleeding piles, } \\
\text { leukoderma, biliousness, } \\
\text { diabetes, skin diseases }\end{array}$ \\
\hline Acacia nilotica Wild. & Mimosaceae & Karuvelai & Tree & $\begin{array}{l}\text { Leaf } \\
\text { Stem bark }\end{array}$ & $\begin{array}{l}\text { Antimutagenic anticancer, } \\
\text { diarrhea, anti-inflammatory } \\
\text { Leukoderma, smallpox, skin } \\
\text { diseases, dysentery, seminal } \\
\text { weakness, biliousness }\end{array}$ \\
\hline Acalypha indica $\mathrm{L}$. & Euphorbiaceae & Kuppaimeni & $\begin{array}{l}\text { Erect } \\
\text { herb }\end{array}$ & $\begin{array}{l}\text { Seed } \\
\text { Whole plant } \\
\text { Leaf }\end{array}$ & $\begin{array}{l}\text { Spasmogenic, antiplasmodia } \\
\text { Asthma, bronchitis, } \\
\text { pneumonia, rheumatism } \\
\text { Ulcer }\end{array}$ \\
\hline Acanthospermum hispidum DC. & Asteraceae & Kaantimull & Herb & $\begin{array}{l}\text { Leaf } \\
\text { Whole plant }\end{array}$ & $\begin{array}{l}\text { Jaundice, malaria, } \\
\text { blennorrhea, hepatobiliary } \\
\text { disorder }\end{array}$ \\
\hline Achyranthes aspera L. & Amaranthaceae & Naiyuruvi & Herb & Whole plant & $\begin{array}{l}\text { Asthmatic cough, snakebite, } \\
\text { piles, abdominal pain, } \\
\text { bronchitis, rabies, influenza, } \\
\text { gonorrhea }\end{array}$ \\
\hline Aegle marmelos Corr. & Rutaceae & Vilvam & Tree & Fruit & Diarrhea, dysentery, piles \\
\hline Aerva lanata Juss. & Amaranthaceae & Poolaipoo, cirupoolai & $\begin{array}{l}\text { Under } \\
\text { shrub }\end{array}$ & Root & Kidney disorder, gonorrhea \\
\hline Agave americana L. & Taxaceae & Kanthaalai & Herb & Leaf & $\begin{array}{l}\text { Dysentery, gonorrhea, } \\
\text { wound, jaundice, snakebite, } \\
\text { hydrophobia }\end{array}$ \\
\hline Allmania nodiflora R. Br. & Amaranthaceae & Kumuti keerai & Herb & Whole plant & Stomach pain, dysentery \\
\hline Aloe vera L. & Liliaceae & Kathazhai and Kumari & Herb & Whole plant & $\begin{array}{l}\text { Skin diseases, menstrual } \\
\text { disorders }\end{array}$ \\
\hline Alternanthera pungens Kunth. & Amaranthaceae & Thevidimullu & Herb & Whole plant & Diuretic, gonorrhea \\
\hline Alternanthera sessilis, R. Br. & Amaranthaceae & $\begin{array}{l}\text { Ponnanganni } \\
\text { keerai }\end{array}$ & Herb & Whole plant & $\begin{array}{l}\text { Hepatitis, bronchitis, asthma, } \\
\text { lung troubles }\end{array}$ \\
\hline Alternanthera triandra Lam. & Amaranthaceae & $\begin{array}{l}\text { Ponnanganni } \\
\text { keerai }\end{array}$ & Herb & Whole plant & Eye trouble \\
\hline Alysicarpus rugosus Dc. & Papilionaceae & Chain pea, red moneywort & Herb & $\begin{array}{l}\text { Root } \\
\text { Seed }\end{array}$ & $\begin{array}{l}\text { Cough } \\
\text { Dysentery, colic swelling }\end{array}$ \\
\hline Amaranthus polygamus L. & Amaranthaceae & Mullukurai & Herb & Seed & Aphrodisiac \\
\hline Amaranthus polygonoides Roxb. & Amaranthaceae & Sirukeerai & Herb & $\begin{array}{l}\text { Leaf } \\
\text { Root }\end{array}$ & $\begin{array}{l}\text { Laxative } \\
\text { Fever, Urinary trouble, } \\
\text { diarrhea, dysentery }\end{array}$ \\
\hline Amaranthus viridis $\mathrm{L}$. & Amaranthaceae & Kuppai keerai & Herb & Whole plant & $\begin{array}{l}\text { Emollient, inflammation } \\
\text { gonorrhea, orchitis, } \\
\text { hemorrhoids } \\
\text { Eve infection }\end{array}$ \\
\hline Andrographis paniculata Nees. & Acanthaceae & Siriyanangai & Herb & Whole plant & $\begin{array}{l}\text { Cancer, diabetes, high blood } \\
\text { pressure, ulcer, leprosy, } \\
\text { bronchitis, skin diseases, } \\
\text { colic, influenza, dysentery, } \\
\text { dyspepsia, snakebite }\end{array}$ \\
\hline Anisomeles malabarica R.Br. & Lamiaceae & Payemiratti & Herb & Whole plant & $\begin{array}{l}\text { Anti-anaphylactic, } \\
\text { antiepileptic, antipyretic, } \\
\text { antispasmodic }\end{array}$ \\
\hline Annona squamosa L. & Anonnaceae & Sita & Tree & $\begin{array}{l}\text { Bark, } \\
\text { Root }\end{array}$ & $\begin{array}{l}\text { Diarrhea } \\
\text { Dysentery }\end{array}$ \\
\hline Asparagus racemosus Willd. & Liliaceae & Nirvekea & Climber & Root & $\begin{array}{l}\text { Nervous disorder, throat } \\
\text { infection, bronchitis }\end{array}$ \\
\hline Asystasia gangetica T. And. & Acanthaceae & Parchorri & Herb & $\begin{array}{l}\text { Leaf } \\
\text { Flower } \\
\text { Whole plant }\end{array}$ & $\begin{array}{l}\text { Anti-inflammatory, asthma } \\
\text { Intestinal astringent } \\
\text { Rheumatic joint }\end{array}$ \\
\hline Azadirachta indica A. Juss. & Meliaceae & Vepamaram & Tree & Whole plant & $\begin{array}{l}\text { Skin diseases, improve liver } \\
\text { function, dental treatment, } \\
\text { urinary infection, intestinal } \\
\text { worms }\end{array}$ \\
\hline
\end{tabular}


Table: 1 (Continued)

\begin{tabular}{|c|c|c|c|c|c|}
\hline Botanical name & Family name & Local name & Habit & Parts used & Medicinal uses \\
\hline Barleria acuminata $\mathrm{W}$. & Acanthaceae & Vellaikurinji & Shrub & Whole plant & $\begin{array}{l}\text { Antimicrobial, } \\
\text { chemotherapeutic, toothache }\end{array}$ \\
\hline \multirow[t]{2}{*}{ Barleria buxifolia L. } & \multirow[t]{2}{*}{ Acanthaceae } & $\begin{array}{l}\text { Kattimullu and } \\
\text { Rosmullippuntu }\end{array}$ & \multirow[t]{2}{*}{ Shrub } & Leaf & $\begin{array}{l}\text { Diaphoretic, expectorant, } \\
\text { catarrhal infection, } \\
\text { anti-inflammatory }\end{array}$ \\
\hline & & & & Seed & Antidote \\
\hline Barleria cuspidata Heyne. & Acanthaceae & Vellaimulli & Shrub & Whole plant & Prevent maceration, cracking \\
\hline Boerhaavia verticillata Poir. & Nyctaginaceae & $\begin{array}{l}\text { Sharunnai, mukkarattai } \\
\text { keerai }\end{array}$ & Herb & Root & Leukorrhea, gonorrhea \\
\hline Borreria hispida K. Sch. & Rubiaceae & Nathaichoori & Shrub & $\begin{array}{l}\text { Leaf } \\
\text { Seed }\end{array}$ & $\begin{array}{l}\text { Astringent, hemorrhoids } \\
\text { Diarrhea, dysentery }\end{array}$ \\
\hline Calotropis gigantea R.Br. & Asclepiadaceae & Erukku & Herb & Whole plant & $\begin{array}{l}\text { Fever, rheumatism, } \\
\text { indigestion, eczema, asthma, } \\
\text { nausea, diarrhea }\end{array}$ \\
\hline Caralluma umbellata Haw. & Asclepiadaceae & Eluman, elumanpuli & Herb & Stem & $\begin{array}{l}\text { Stomach disorder, abdominal } \\
\text { pain, obesity, ulcer }\end{array}$ \\
\hline Cardiospermum halicacabum L. & Sapindaceae & Mudakathan keerai & Herb & Whole plant & $\begin{array}{l}\text { Anti-inflammation, diuretic, } \\
\text { laxative, analgesic, stomachic }\end{array}$ \\
\hline Cassia auriculata L. & Caesalpiniaceae & Aavaram & Tree & Whole plant & $\begin{array}{l}\text { Diabetes, joint and muscle } \\
\text { pain (rheumatism), eye } \\
\text { infections (conjunctivitis), } \\
\text { constipation, liver disease, } \\
\text { urinary tract diseases, } \\
\text { jaundice }\end{array}$ \\
\hline Catharanthus roseus (L.) G.Don. & Apocynaceae & Nithya kalyani & Herb & Whole plant & Leukemia, cancer \\
\hline Celosia argentea $\mathrm{L}$. & Amaranthaceae & Kozhi poo & Shrub & $\begin{array}{l}\text { Whole plant } \\
\text { Seed }\end{array}$ & $\begin{array}{l}\text { Wound, sores, skin eruption } \\
\text { Jaundice, gonorrhea, wound, } \\
\text { fever }\end{array}$ \\
\hline Celosia polygonoides Retz. & Amaranthaceae & Pannai keerai & Herb & Whole plant & Insect bite, skin disease \\
\hline Chloris barbata Sw. & Poaceae & Kodaipillu, sevarugu pillu & Herb & Whole plant & $\begin{array}{l}\text { Rheumatism, skin disorder, } \\
\text { antidiabetic, antibacterial, } \\
\text { antimicrobial }\end{array}$ \\
\hline Cissus quadrangularis $\mathrm{L}$. & Vitaceae & Pirandai & Shrub & Whole plant & $\begin{array}{l}\text { Obesity, diabetes, heart } \\
\text { diseases, high cholesterol, } \\
\text { indigestion, piles }\end{array}$ \\
\hline Cissus repanda Vahl. & Vitaceae & $\begin{array}{l}\text { Chunnam buvalli, } \\
\text { nerrinampulli }\end{array}$ & Climber & Whole plant & Bone fracture, wound \\
\hline \multirow[t]{2}{*}{ Citrullus colocynthis Schrad. } & \multirow[t]{2}{*}{ Cucurbitaceae } & \multirow[t]{2}{*}{ Pikkumutti } & \multirow[t]{2}{*}{ Herb } & Fruit & $\begin{array}{l}\text { Purgative, anthelmintic, } \\
\text { antipyretic, carminative, } \\
\text { leukoderma, ulcer, urinary } \\
\text { diseases, leukoderma, asthma, } \\
\text { bronchitis, anemia }\end{array}$ \\
\hline & & & & Root & $\begin{array}{l}\text { Rheumatism, inflammation, } \\
\text { pimples }\end{array}$ \\
\hline Cleome felina L.F. & Capparidaceae & Asagandar & Herb & Whole plant & Neuromuscular \\
\hline Cleome viscosa $\mathrm{L}$. & Capparidaceae & Naikkaduku & Herb & Whole plant & $\begin{array}{l}\text { Ringworm, dyspepsia, } \\
\text { flatulence, bronchitis }\end{array}$ \\
\hline \multirow[t]{2}{*}{ Clitoria ternatea $\mathrm{L}}$. & \multirow[t]{2}{*}{ Fabaceae } & \multirow[t]{2}{*}{ Sangupu, karka karutan } & \multirow[t]{2}{*}{ Climber } & $\begin{array}{l}\text { Leaf, seed } \\
\text { Whole plant }\end{array}$ & $\begin{array}{l}\text { Rheumatism, rubefacient, } \\
\text { Antipyretic, diuretic, } \\
\text { anthelmintic, leprosy, } \\
\text { inflammation, leukoderma, } \\
\text { bronchitis, asthma }\end{array}$ \\
\hline & & & & Seed & Cathartic \\
\hline Coccinia indica $\mathrm{W} \& \mathrm{~A}$ & Cucurbitaceae & Kovai & Climber & Whole plant & $\begin{array}{l}\text { Gastrointestinal problems, } \\
\text { blood purification, asthma, } \\
\text { bronchitis }\end{array}$ \\
\hline \multirow[t]{2}{*}{ Cocculus hirsutus DC. } & \multirow[t]{2}{*}{ Menispermaceae } & \multirow[t]{2}{*}{ Kattukodi } & \multirow[t]{2}{*}{ Herb } & Whole plant & $\begin{array}{l}\text { Diuretic, stomach ache, } \\
\text { laxative, Night blindness, }\end{array}$ \\
\hline & & & & Leaf & $\begin{array}{l}\text { Skin infection, eczema, } \\
\text { rheumatism, gonorrhea } \\
\text { laxative, diuretic, fever }\end{array}$ \\
\hline
\end{tabular}


Table: 1 (Continued)

\begin{tabular}{|c|c|c|c|c|c|}
\hline Botanical name & Family name & Local name & Habit & Parts used & Medicinal uses \\
\hline \multirow[t]{2}{*}{ Commelina benghalensis $\mathrm{L}$. } & Commelinaceae & Aduthinna thalai & Herb & Whole plant & $\begin{array}{l}\text { Skin diseases, astringent, } \\
\text { demulcent, laxative, eye } \\
\text { complaints }\end{array}$ \\
\hline & & & & Root & Stomach disorder \\
\hline \multirow[t]{2}{*}{ Commiphora caudata Engl. } & Burseraceae & Mangiluvai & $\begin{array}{l}\text { Small } \\
\text { Tree }\end{array}$ & Leaf & $\begin{array}{l}\text { Antipasmodic, cytotoxic, } \\
\text { hypothermic }\end{array}$ \\
\hline & & & & Seed & Stomach ache \\
\hline Corchorus aestuans L. & Tiliaceae & Perumpinak kukirai & Herb & $\begin{array}{l}\text { Whole plant } \\
\text { Seed }\end{array}$ & $\begin{array}{l}\text { Stomach ache, pneumonia } \\
\text { Carminative, febrifuge }\end{array}$ \\
\hline Corchorus trilocularis, L. & Tiliaceae & $\begin{array}{l}\text { Talakkaip } \\
\text { poondu }\end{array}$ & Herb & Whole plant & Anti-inflammatory \\
\hline Crotalaria ovalifolia Wall. & Papilionaceae & - & Herb & Whole plant & Antipyretic \\
\hline \multirow[t]{3}{*}{ Crotalaria retusa $\mathrm{L}}$. & Fabaceae & Kilukiluppai & Herb & Whole plant & Skin infection \\
\hline & & & & Leaf $r$ raf & Scabies, lung diseases \\
\hline & & & & Seed & Snakebite \\
\hline Croton sparsiflorus Mor. & Euphorbiaceae & Aathupoondu & Herb & Whole plant & $\begin{array}{l}\text { Hypotensive, } \\
\text { anti-inflammatory, antipyretic, } \\
\text { antibacterial, antifungal }\end{array}$ \\
\hline Cuscuta chinensiis Lam. & Convolvulaceae & Amar bel & $\begin{array}{l}\text { Parasitic } \\
\text { twiner }\end{array}$ & Whole plant & Kidney deficiency \\
\hline Cyperus rotundus $\mathrm{L}$. & Cyperaceae & Korai, koraikilangu & Herb & Whole plant & $\begin{array}{l}\text { Menstrual irregularities, } \\
\text { fever, diarrhea, dysentery, } \\
\text { emmenagogue, intestinal } \\
\text { problem, wound, stomach } \\
\text { ailments }\end{array}$ \\
\hline Datura metal L. & Solanaceae & Vellaiumathai & Herb & Whole plant & $\begin{array}{l}\text { Antiasthma, antispasmodic, } \\
\text { hypnotic, narcotic }\end{array}$ \\
\hline Desmodium triflorum DC. & Fabaceae & Cirupullati & Herb & Whole plant & Ulcer, skin disease, wound \\
\hline \multirow[t]{2}{*}{ Dichrostachys cinerea W. \& A. } & Mimosaceae & Veduttalam & Thorny & Bark & Dysentery \\
\hline & & & Shrub & Root & $\begin{array}{l}\text { Anthelmintic, purgative, } \\
\text { diuretic, laxative, bone } \\
\text { fracture }\end{array}$ \\
\hline \multirow[t]{2}{*}{ Digera arvensis Forsk. } & Amaranthaceae & Toyyak kirasi & Herb & Whole plant & Renal disorder \\
\hline & & & & Flower seed & Urinary disorder \\
\hline \multirow[t]{2}{*}{ Diospyros ebenum Koen. } & Ebenaceae & Acha, karunkali & Tree & Fruit & Attenuant, lithontripic \\
\hline & & & & Bark & Cough, asthma, diabetes \\
\hline \multirow[t]{3}{*}{ Eclipta alba Hassk. } & Compositae & Karisilaan ganni & Herb & Whole plant & $\begin{array}{l}\text { Ringworm, spleen, liver } \\
\text { enlargement, jaundice, } \\
\text { hepatitis, wound }\end{array}$ \\
\hline & & & & Root & Purgative, emetic \\
\hline & & & & Leaf & Scorpion sting, anthelmintic \\
\hline Euphorbia hirta L. & Euphorbiaceae & Amman pacarisi & Herb & Whole plant & $\begin{array}{l}\text { Cough, bronchitis, asthma, } \\
\text { jaundice, dysentery, tumors, } \\
\text { gonorrhea }\end{array}$ \\
\hline Euphorbia tirucalli L. & Euphorbiaceae & Catukalli, kalli & Herb & Whole plant & $\begin{array}{l}\text { Cancer, asthma, cough, } \\
\text { neuralgia, rheumatism }\end{array}$ \\
\hline Evolvulus alsinoides L. & Convolvulaceae & Vishnu kranthi & Herb & Whole plant & $\begin{array}{l}\text { Febrifuge, loss of memory, } \\
\text { fever nervous debility, } \\
\text { dysentery }\end{array}$ \\
\hline Ficus bengalensis L. & Moraceae & Aal, peral & Tree & Bark, leaf & $\begin{array}{l}\text { Skin diseases, chronic } \\
\text { diarrhea, dysentry }\end{array}$ \\
\hline Ficus racemosa $\mathrm{L}$. & Moraceae & Atti & Tree & Whole plant & $\begin{array}{l}\text { Diabetes, diarrhea, } \\
\text { inflammation, respiratory, } \\
\text { urinary diseases }\end{array}$ \\
\hline Ficus religiosa $\mathrm{L}$. & Moraceae & Arasu & Tree & Whole plant & $\begin{array}{l}\text { Diabetes, diarrhea, asthma, } \\
\text { epilepsy, inflammation, gastric } \\
\text { problems }\end{array}$ \\
\hline Gloriosa superba L. & Liliaceae & $\begin{array}{l}\text { Kanthal malar, kanvilli, kalapi } \\
\text { kilangu }\end{array}$ & Climber & Whole plant & $\begin{array}{l}\text { inflammation, rheumatism, } \\
\text { ulcer, leprosy, snakebite, } \\
\text { gonorrhea, abdominal pain, } \\
\text { cancer, itching, purgative }\end{array}$ \\
\hline Gomphrena decumbens Jacq. & Amaranthaceae & Arasa con todo & Herb & Whole plant & $\begin{array}{l}\text { Antibacterial, antimalarial, } \\
\text { diuretic }\end{array}$ \\
\hline Gynandropis pentaphylla DC. & Capparidaceae & Naalvalai & Herb & Whole plant & Anti-inflammatory \\
\hline Hibiscus vitifolius $\mathrm{L}$. & Malvaceae & Manithuthi & Herb & Root bark & $\begin{array}{l}\text { Jaundice, inflammation } \\
\text { diabetes }\end{array}$ \\
\hline Indigofera trita L. f. & Fabaceae & Punal murunkai, kattauvari & Shrub & Whole plant & $\begin{array}{l}\text { Nematicide, scorpion bite, } \\
\text { stomach cancer }\end{array}$ \\
\hline
\end{tabular}


Table: 1 (Continued)

\begin{tabular}{|c|c|c|c|c|c|}
\hline Botanical name & Family name & Local name & Habit & Parts used & Medicinal uses \\
\hline Ipomaea aquatic Forsk. & Convolvulaceae & Nali, nalikam, vallai & Herb & Leaf & Green leafy vegetable \\
\hline Jasminum angustifolium Vahl. & Oleaceae & $\begin{array}{l}\text { Pithikam, pichi poo, kaatum } \\
\text { alligai }\end{array}$ & $\begin{array}{l}\text { climbing } \\
\text { shrub }\end{array}$ & Leaf & Emetic \\
\hline Jatropha curca, L. & Euphorbiaceae & Katta manakku & Herb & Whole plant & $\begin{array}{l}\text { Insecticide, fungicide, } \\
\text { antitumor, wound }\end{array}$ \\
\hline Kyllinga triceps Rottb. & Cyperaceae & Velutta nirbasi & Herb & $\begin{array}{l}\text { Root } \\
\text { Whole plant }\end{array}$ & $\begin{array}{l}\text { Diabetic, diuretic, demulcent } \\
\text { Antipyretic }\end{array}$ \\
\hline Leonotis nepetaefolia R.Br. & Lamiaceae & Irana-peri & Tall herb & Whole plant & $\begin{array}{l}\text { Diuretic, diarrhea, } \\
\text { gastrointestinal trouble }\end{array}$ \\
\hline Leucas aspera Spr. & Lamiaceae & Thumbai & Herb & $\begin{array}{l}\text { Whole plant } \\
\text { Leaf }\end{array}$ & $\begin{array}{l}\text { Antipyretic, insecticide } \\
\text { Chronic rheumatism, } \\
\text { snakebites }\end{array}$ \\
\hline Lippia nodiflora Mich. & Verbenaceae & Poduthu valai & Herb & Leaf, fruit & $\begin{array}{l}\text { Diarrhea, gonorrhea, bleeding } \\
\text { piles }\end{array}$ \\
\hline Loranthus longiflorus Desv. & Loranthaceae & Pulluri & $\begin{array}{l}\text { Stem } \\
\text { parasitic }\end{array}$ & Whole plant & Antifertility, anticancer, \\
\hline Ludwigia perennis $\mathrm{L}$. & Ongaraceae & $\begin{array}{l}\text { Neer karayambu, musal } \\
\text { kithilai }\end{array}$ & $\begin{array}{l}\text { shrub } \\
\text { Herb }\end{array}$ & $\begin{array}{l}\text { Leaf } \\
\text { Leaf } \\
\text { Root }\end{array}$ & $\begin{array}{l}\text { Skin diseases } \\
\text { Skin diseases } \\
\text { Cancer }\end{array}$ \\
\hline Martynia annua L. & Pedaliaceae & Puli-Nagam & Herb & Whole plant & $\begin{array}{l}\text { Antiepileptic, antiseptic, } \\
\text { wounds }\end{array}$ \\
\hline Melia azedarach L. & Meliaceae & Malaivembu & Tree & $\begin{array}{l}\text { Leaf } \\
\text { Root }\end{array}$ & $\begin{array}{l}\text { Leprosy, anthelmintic, diuretic } \\
\text { Ulcer, rheumatism, skin } \\
\text { diseases, ringworm, scabies }\end{array}$ \\
\hline $\begin{array}{l}\text { Micrococca mercurialis (L.) } \\
\text { Benth. }\end{array}$ & Euphorbiaceae & Kunuk kuththukki & Herb & Leaf & Purgative \\
\hline Mimosa pudica L. & Mimosaceae & Thotta chinungi & Herb & Root & Muscular pain, piles \\
\hline Mollugo cerviana Ser. & Aizoaceae & Parpadagam, tura & Herb & Whole plant & Fever, thirst, diarrhea \\
\hline Mollugo nudicaulis Lam. & Aizoaceae & $\begin{array}{l}\text { Parpadagam, } \\
\text { ullukumarannuchedi }\end{array}$ & Herb & Whole plant & $\begin{array}{l}\text { Cough, jaundice, wound } \\
\text { healing, diabetes, ringworm }\end{array}$ \\
\hline Mollugo pentaphylla L. & Aizoaceae & Siruserupati & Herb & Whole plant & Anti-inflammatory, antipyretic \\
\hline Orygia decumbens Forsk. & Aizoaceae & Numnel likeeray & Herb & Whole plant & Kidney stone, gonorrhea \\
\hline Oxalis corniculata L. & Oxalidaceae & Pulliyaarai & Herb & Leaf & Stomach pain \\
\hline Passiflora foetida $\mathrm{L}$. & Passifloraceae & $\begin{array}{l}\text { Mupparisavalli, sirupu } \\
\text { naikalli }\end{array}$ & Herb & Whole plant & $\begin{array}{l}\text { Digestive problem, dyspepsia, } \\
\text { diarrhea, astringent }\end{array}$ \\
\hline Pedalium murex L. & Pedaliaceae & Anainerunji & Herb & Whole plant & $\begin{array}{l}\text { Digestive, carminative, } \\
\text { spasmodic, inflammation, } \\
\text { flatulence, kidney stone }\end{array}$ \\
\hline $\begin{array}{l}\text { Phyllanthus } \\
\text { emblica L. }\end{array}$ & Euphorbiaceae & Nelli & Tree & Fruit & $\begin{array}{l}\text { Antioxidant, antitumor, } \\
\text { anticarcinogenic }\end{array}$ \\
\hline Phyllanthus maderaspatensis L. & Euphorbiaceae & Melanelli & Tree & Leaf & Purgative, rheumatism \\
\hline & & & & $\begin{array}{l}\text { Seed } \\
\text { Whole plant }\end{array}$ & $\begin{array}{l}\text { Carminative, diuretic, laxative } \\
\text { Bronchitis, jaundice }\end{array}$ \\
\hline Phyllanthus niruri, L. & Euphorbiaceae & Keezhanelli & Tree & Whole plant & $\begin{array}{l}\text { Astringent, laxative, } \\
\text { carminative, gonorrhea, } \\
\text { dyspepsia, urinary disorders, } \\
\text { jaundice, diuretic }\end{array}$ \\
\hline Polygala rosmarinifolia W.\&A. & Polygalaceae & - & Herb & Whole plant & Snakebite, cough, bronchitis \\
\hline Pongamia glabra Vent. & Fabaceae & Punga maram & Tree & Bark & Skin disease \\
\hline Portulaca oleracea L. & Portulacaceae & keerai & Herb & Whole parts & Kidney problem \\
\hline Portulaca quadrifida L. & Portulacaceae & Pasalai keerai & Herb & Whole plant & $\begin{array}{l}\text { Rheumatism, diuretic, ulcer, } \\
\text { eczema, dermatitis, dysentery }\end{array}$ \\
\hline Ruellia punctata Nees. & Acanthaceae & Krishikorsh & Shrub & Whole plant & Malaria \\
\hline Sapindus emarginatus Vahl & Sapindaceae & Ponankottai, poovandi kottai & Tree & Whole plant & Asthma, colic, dysentery \\
\hline $\begin{array}{l}\text { Sarcostemma brevistigma W. } \\
\text { \& A. }\end{array}$ & Asclepiadaceae & Kondapala, kodikalli & $\begin{array}{l}\text { Trailing } \\
\text { shrub }\end{array}$ & Whole plant & $\begin{array}{l}\text { Asthma, rheumatism, joint } \\
\text { pain, chronic ulcer, bronchitis, } \\
\text { snakebite }\end{array}$ \\
\hline Sida acuta Burm. & Malvaceae & Palambasi & Herb & Whole plant & Asthma, ulcer, snakebite \\
\hline Sida cordifolia $\mathrm{L}$. & Malvaceae & Mayil-manika & Herb & Whole plant & $\begin{array}{l}\text { Fat loss, asthma, heart } \\
\text { diseases }\end{array}$ \\
\hline Solanum pubescens Willd. & Solanaceae & Kaatu sundaikkai & $\begin{array}{l}\text { Under } \\
\text { shrub }\end{array}$ & Whole plant & Joint pain, analgesic activity \\
\hline
\end{tabular}


Table: 1 (Continued)

\begin{tabular}{|c|c|c|c|c|c|}
\hline Botanical name & Family name & Local name & Habit & Parts used & Medicinal uses \\
\hline Solanum torvum Sw. & Solanaceae & Sundaikkai & Shrub & $\begin{array}{l}\text { Leaf } \\
\text { Whole plant }\end{array}$ & $\begin{array}{l}\text { Wound, skin diseases } \\
\text { Asthma, rheumatism, dropsy, } \\
\text { gonorrhea }\end{array}$ \\
\hline Tectona grandis L. f. & Verbenaceae & Tekku & Tree & Whole plant & $\begin{array}{l}\text { Bronchitis, dysentery, } \\
\text { diabetes, leprosy }\end{array}$ \\
\hline Tephrosia purpurea Pers. & Fabaceae & Kollukkai & Herb & Whole plant & $\begin{array}{l}\text { Ulcer, asthma, spleen, liver } \\
\text { disease, leprosy, anthelmintic }\end{array}$ \\
\hline Thevetia neriifolia Juss. & Apocynaceae & Yellow oleander & Shrub & Whole plant & $\begin{array}{l}\text { Leprosy, eye diseases, skin } \\
\text { disorders }\end{array}$ \\
\hline Tinospora cordifolia Miers. & Menispermaceae & Gunduchi & Herb & Whole plant & $\begin{array}{l}\text { Diabetes, cancer, rheumatoid } \\
\text { arthritis, peptic ulcer disease, } \\
\text { fever, gonorrhea }\end{array}$ \\
\hline Trianthema portulacastrum L. & Aizoaceae & Saaranatthi & Herb & Whole plant & Disease resistance \\
\hline Trichodesma indicum (L.) R. Br. & Boraginaceae & Kallutaitumpai & Herb & Whole plant & Skin disease, snakebite \\
\hline Tridax procumbens L. & Asteraceae & Vettu kayathalai & Herb & Whole plant & $\begin{array}{l}\text { Wound healing, antifungal, } \\
\text { insect repellent }\end{array}$ \\
\hline Vitex negundo L. & Verbenaceae & Nochi & Shrub & Leaf & $\begin{array}{l}\text { Fever, chronic, intestinal } \\
\text { worm, spleen disorder }\end{array}$ \\
\hline Waltheria indica $\mathrm{L}$. & Sterculiaceae & Shengali poondu & Shrub & Whole plant & $\begin{array}{l}\text { Febrifuge, fever, cold, bladder } \\
\text { ailment, vaginal infection, } \\
\text { wound healing }\end{array}$ \\
\hline Wrightia tinctoria R.Br. & Apocynaceae & Nilapalai & Tree & Whole plant & $\begin{array}{l}\text { Fever, skin disorder, } \\
\text { anti-dandruff, hair oil }\end{array}$ \\
\hline Zizyphus jujuba Lam. & Rhamnaceae & Illanthai & Tree & Whole plant & Diarrhea, mental retardation \\
\hline Ziziphus oenoplia Mill. & Rhamnaceae & Suraimullu & $\begin{array}{l}\text { Small } \\
\text { tree }\end{array}$ & Whole plant & Wound healing, astringent \\
\hline $\begin{array}{l}\text { Actiniopteris radiata (J. Koenig. } \\
\text { ex Sw.) Link }\end{array}$ & Actinopteridaceae & Small fern & & Whole plant & Asthma, cough \\
\hline
\end{tabular}

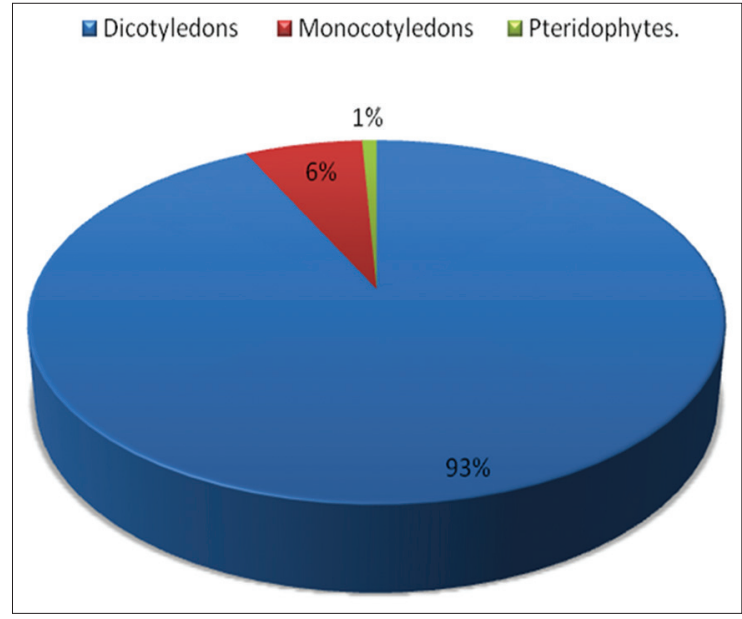

Fig. 1: Percentage of the classification of plants

worms, urinary tract diseases, antimicrobial, chemotherapeutic, diaphoretic, expectorant, antidote, heart diseases, anemia, hernia, leukorrhea, indigestion, eczema, nausea, hemorrhoids, liver disease, wound, purgative, anthelmintic, antipyretic, carminative, leukoderma, dyspepsia, night blindness, pneumonia febrifuge, scabies, spleen, liver enlargement, anticarcinogenic, and mental retardation (Table 1).

People of the study area mostly administered phytomedicine orally. A majority of remedies are prepared in the form of extract or juice followed by powder form, decoction, and freshly collected plant parts. Most of the villagers in the study are poor, and they largely depend on plants for food, medicine, fuel, and other daily necessities.

\section{DISCUSSION}

Ranganathan et al. [10] assured that ethnobotanical survey was made on the utilization of medicinal plants among the people of selected six villages

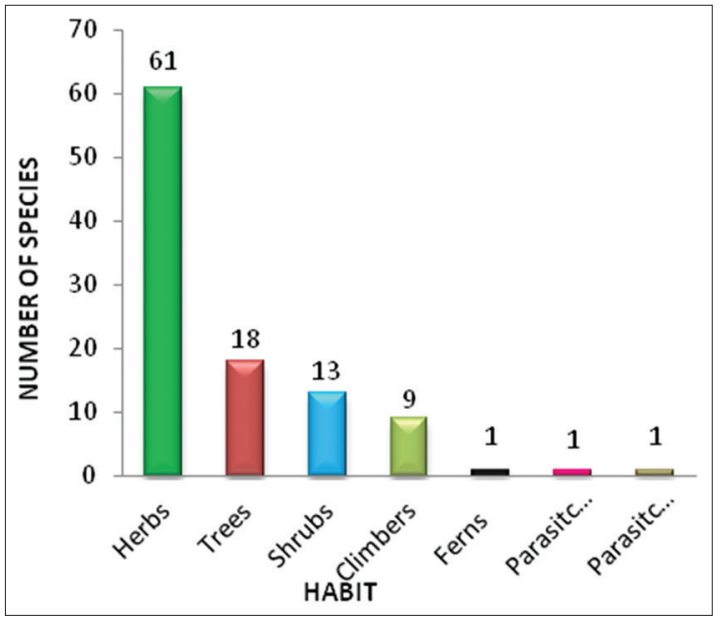

Fig. 2: Percentage of medicinal plants on the basis of their habit

from Jawadhu Hills in Tamil Nadu, which was carried out during December 2009-April 2010. They use forest plants, weeds, fruit plants, vegetables, spices, ornamental plants, ferns, and many others as traditional medicine. Although many of these species are known as medicinal plants, others are mainly used for non-medicinal purposes such as preparing agricultural implements. Santalum album, Terminalia bellirica, Cassia fistula, Gymnema sylvestre, Melia dubia, and Rauvolfia tetraphylla are the leading species used as remedies against a variety of complaints. The results of the present study provide evidence that medicinal plants continue to play an important role in the health-care system of this tribal (Malayalis) community in Jawadhu Hills of Tami Nadu.

The indigenous knowledge on medicinal plants is gaining recognition worldwide because of its support in the discovery of new medicines and its importance for proper conservation of biodiversity. This paper documents the traditional knowledge of medicinal plants used for the 
treatment of skin diseases by the tribe, namely, Kaani, of Kanyakumari District, Tamil Nadu, India. The present study was done through structured questionnaires in consultation with the tribal ethnomedical practitioners and has resulted in the documentation of 55 medicinal plant species belonging to 38 families. For curing the skin disease, the use of aboveground plant parts was higher $(83.33 \%)$ than the underground plant parts (16.67\%). Of the aboveground plant parts, leaves were used in the majority of cases (19 species), followed by whole plants (11 species). Different underground plant forms such as roots and rhizomes were also used by the tribe as medicine. Johnsy et al. [11] thus underlined the potentials of the ethnobotanical research and the need for the documentation of traditional ecological knowledge pertaining to the medicinal plant utilization for the greater benefit of humankind.

Ethnobotanical study to investigate the ethnomedicinal uses of plants by the Batswapong tribe, Eastern Botswana, was done. This revealed a wealth of traditional knowledge on uses of medicinal plants. 36 plants distributed across 22 families were recorded to treat 60 ailments. Most of these plants were trees (61\%) with roots $(82 \%)$ being the most frequently used parts for the preparation of remedies across the 22 families. For each species, its botanical family and vernacular name, medicinal uses, parts used, and mode of preparation were documented. This study has revealed that knowledge on uses of medicinal plants is shrinking because of restrictions from religions, migration to urban areas, and lack of interest by younger generations on uses of medicinal plants. Motlhanka and Nthoiwa [12] concluded by advocating for the implementation of government policies that will significantly contribute toward the preservation of biodiversity and indigenous traditional knowledge of medicinal flora.

Ethnobotanical survey was conducted in the remote hills, forests, and rural areas of Tripura, a diversified ethnic people rich state of Northeastern India, for gathering information about the traditional method of birth control [13]. A semi-structured questionnaire was used during the interview with the informants having traditional botanical knowledge. The use of medicinal plants was documented using an interview datasheet mentioning the detailed information of the informants and vernacular names, parts used, method of preparation, and administration modes of botanicals. Finally, collected samples of botanicals, prepared herbarium, and identified and scientific names were confirmed by consulting reference herbarium specimen available in Assam University, Silchar. A total of 55 ethnomedicinal plants belonging to 42 families and 49 genera have been documented having antifertility property. Apocynaceae, Caesalpiniaceae, Combretaceae, and Fabaceae were found to be the dominant families of medicinal plants used for fertility regulation. This paper represents the detailed profile of each plant including scientific name, family, common name, parts used, activities, mode of preparation, and dosage. While comparing the established literature, it is interestingly recorded that antifertility activity of 10 plants has been reported for the first time. Conservation of the traditional informations should be given utmost importance in this region to prevent the rapid loss of ethnobotanical wealth.

Women in Katsina State, Nigeria, have been using medicinal plants to cure various ailments associated with maternal health since time immemorial; however, the use of such plants was never documented. In this study, an ethnobotanical survey was conducted to document medicinal plants used for traditional maternal health care in Katsina State, Nigeria [14]. A semistructured questionnaire method was used to interview 300 respondents ( 50 from two local government areas of each of the 3 senatorial districts) comprising of herbalists, traditional birth attendants, traditional medical practitioners, house wives, farmers, and others. Medicinal plants belonging to 101 genera distributed among 50 families were documented. Most of the reported plants belong to the Fabaceae (22.52\%), Asteraceae (7.21\%), Malvaceae (5.41\%), and Anacardiaceae (4.51\%) families. Acacia nilotica (L.) Delile and Guiera senegalensis J.F. Gmel had the highest relative frequency of citation and fidelity level of $0.93 ; 100 \%$ and $0.92 ; 100 \%$, respectively. Among the 18 categories of ailments, headache, navel pain, postpartum hemorrhage, and postpartum wound healing had the highest informant consensus factor of 1.00 each. Most of the reported plants $(68.47 \%)$ were herbs and shrubs, and about $84.68 \%$ of the surveyed plants were wild. Leaves were the most frequently used (32.14\%) plant's part. Most of the medications (32\%) were prepared as decoctions and preparations are mostly administered orally (84.68\%). Scientific validation of the biological properties of the surveyed plants is highly advocated, and cultivation of medicinal plants to minimize the pressure on wild species is also recommended.

Kodi et al. [15] identified 33 plant species from 30 genera that consisted of Flueggea virosa (Willd.) Voigt, Securidaca longipedunculata, Erythrina abyssinica, Melia azedarach, Carissa edulis Fork, Harrisonia abyssinica Olive., Zanthoxylum chalybeum Engl., Psidium guajava, Citrus sinensis, Schkuhria pinnata (Lam.), Lantana camara, Carica papaya, Mangifera indica L., Azadirachta indica., Persea americana Mill., Bidens pilosa, Cymbopogon citratus (DC), Plectranthus barbatus, Maytenus senegalensis, Citrus reticulata, Ocimum gratissimum, Ocimum basilicum, Croton macrostachyus Olive., Oncoba spinosa Forssk., Steganotaenia araliacea, Acacia sieberiana, Ormocarpum trachycarpum, Acacia hockii De Wild., Euclea latideus Staff, Cassia hirsuta, Chamaecrista nigricans Greene, Butyrospermum paradoxum, and Aristolochia tomentosa (Sims) were identified as being used by communities as antimalarial plants. These belong to 23 families of Rutaceae, Lamiaceae, Euphorbiaceae, Mimosaceae, Celastraceae, Meliaceae, Asteraceae, Papilionaceae, Polygalaceae, Flacourtiaceae, Umbelliferae, Sapotaceae, Apocynaceae, Simaroubaceae, Ebenaceae, Aristolochiaceae, Anacardiaceae, Caricaceae, Lauranceae, Myrtaceae, Verbenaceae, Poaceae, and Celastraceae. The most frequently used medicinal plants were from the families: Rutaceae and Lamiaceae (13.0\% each) that had three plant species each. 10 species $(30.3 \%)$ were identified and documented for the first time in Uganda to treat malaria. The most commonly used plant part was the root $(44.68 \%)$, followed by leaves (38.30\%), stem (6.38\%) each, bark, and whole plant $(4.30 \%)$ each, and the least was the seeds with $2.13 \%$. The growth habits included trees (48.48\%), shrubs and herbs (24.24\% each), and the climbers with the least percentage of $3.03 \%$. The largest habitat of the medicinal plants was found in the homesteads (21.10\%), followed by wooded grassland and garden with $18.20 \%$, grasslands (15.20\%), open grassland $(9.10 \%)$, cultivated and roadside $(6.10 \%$ each), and garden edge and forest had the lowest value of $3.0 \%$ each. Most of the plant materials were dried in the shade, pounded into a powder, and taken orally as water decoctions $(76.50 \%)$ and infusions (23.50\%). Many plants used traditionally for the treatment of malaria were identified, and claims of some of the medicinal plants documented in the survey are supported by literature. However, the scientific validation of the traditional claims of antimalarial activity of some of these plants not researched on is needed. This includes testing for efficacy, safety (toxicity), antiplasmodial screening, and structure elucidation to find the identity of active compounds present. This would make them considered for future research for active compounds and the possible synthesis of new, cheaper, and more effective antimalarial drugs. This would help in conserving and sustainable use of the antimalarial plants. Therefore, it is necessary to carry out research to solve these problems so that the lives of people are not at risk.

Aadhan and Anand [16] reported an ethnobotanical and literature survey to collect information about medicinal plants used for the treatment of diabetics and associated complications by Paliyar tribal people of Sadhuragiri hills. Analysis of remedies obtained from different plant parts was performed. The indigenous knowledge of plants used for the treatment of diabetics was collected through questionnaire and personal interviews. A total of 65 plants used to treat diabetes have been documented. The investigation revealed that leaves (27\%), followed by fruits $(11 \%)$, roots $(6 \%)$, seeds $(6 \%)$, flower $(6 \%)$, bark $(4 \%)$, whole plant $(4 \%)$, tuber $(3 \%)$, rhizomes $(2 \%)$, and bulb $(1 \%)$ were mostly used for the treatment of diabetes. Antidiabetic medicinal plants used by Tamil people have been listed along with plant parts used and its active chemical constituents. 
Structural Abstract

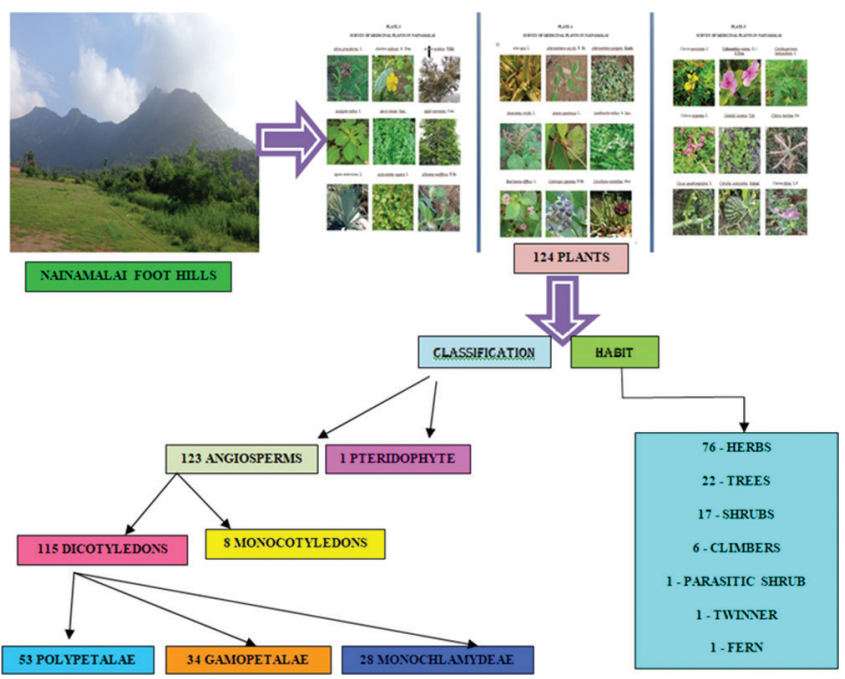

\section{CONCLUSION}

In the present study, plant survey at Ninamalai hill has documented a total of 124 plants. Among 124 species surveyed, 123 were angiospermic plants and one pteridophytic plant. Analysis of plants showed that more number of dicotyledons ( 115 species) belongs to 43 families and 8 monocotyledons are represented in 5 families. Within dicotyledons, 53 species are polypetalae, 34 species are gamopetalae, and 28 are monochlamydeae. For each species, botanical name, family, habit, local name, parts used, and medicinal usage are identified and presented. The family-wise analysis showed that the family Amaranthaceae held the dominant position by representing more number of species (13 species). Habit-wise analysis shows that maximum representatives to the plants are from herb (76) community, followed by trees (22), shrubs (17), climbers (6), parasitic shrub and parasitic twinner (1), and fern (1). The absence of epiphytes indicates the loss special habitats due to the degraded status of the hill. Part-wise analysis showed the predominant use of whole plants followed by leaves, root, seed, bark, fruit, flower, and stem. Herbal remedies play a fundamental role in traditional medicine in some tribal people of rural regions in Tamil Nadu. These collected medicinal plants are used for the treatment of several diseases such as ulcer, anti-inflammatory, anthelmintic, analgesic, leprosy, gonorrhea, bronchitis, diarrhea, dysentery, bleeding piles, leukoderma, diabetes, antimutagenic, antimicrobial, anticancer, smallpox, seminal weakness, biliousness, spasmogenic, antiplasmodia, asthma, pneumonia, rheumatism, jaundice, malaria, blennorrhea, hepatobiliary disorder, asthmatic cough, snakebite, abdominal pain, rabies, influenza, fever, cold, cough, eye diseases, kidney disorder, wound, hydrophobia, stomach pain, menstrual disorders, hepatitis, lung troubles, colic swelling, aphrodisiac, laxative, urinary trouble, emollient, inflammation, orchitis, hemorrhoids, eye infection, cancer, high blood pressure, leprosy, dyspepsia, antianaphylatic, antiepileptic, antipyretic, antispasmodic, nervous disorder, throat infection, anti-inflammatory, asthma, intestinal astringent, rheumatic joint, dental treatment, urinary infection, intestinal worms, joint, muscle pain (rheumatism), eye infections, constipation, liver disease, and urinary tract diseases.

\section{REFERENCES}

1. Uraku AJ, Okaka AN, Ibiam UA, Agbafor KN, Obasi NA, Ajah PM, et al. Antiplasmodial activity of ethanolic leaf extracts of Spilanthes uliginosa, Ocimum basilicu (Sweet Basil), Hyptis spicigera and Cymbopogon citratus on mice exposed to Plasmodium berghei Nk 65. Int J Biochem Res Rev 2015;6:28-36.

2. Pomnha TH. Medicinal Plants and their Importance. Mockba: Professional English for Cultivation and Processing Technology of Medicinal and Essential-oil Plants; 2014.

3. Sujatha G, Mariya SS. Ethnomedicinal survey of flora used by Chettiapatti inhabitants, Manapparai. Asian J Biochem Pharm Res 2015;4:2231-560.

4. Bhuvaneswari R, Ramanathan R, Krishnapriya P, Madheswaran A, Dhandapani R. Survey of wild tuberous medicinal plants of Kolli hills in Namakkal district, Tamil Nadu, India. Int J Herb Med 2015;3:41-8.

5. Pullaiah T, Rao DM. Flora of Eastern Ghats. Vol. 1. New Delhi: Regency Publications; 2002.

6. Jain SK, Rao RR. A Hand Book of field and Herbarium Methods. New Delhi: Today and Tomorrows Publishers; 1976.

7. Rao RR, Sharma BD. A Manual for Herbarium Collections. Calcutta: Botanical Survey of India; 1990. p. 184.

8. Gamble GS, Fischer CF. Flora of presidency of Madras. Vol. 1-3. Calcutta, India: Botanical Survey of India; 1967.

9. Mathew KM. Flora of the Tamil Nadu and Carnatic. Vol. 1. St. Joseph's College, Trichy, Tamil Nadu: The Rapinat Herbarium; 1981.

10. Ranganathan R, Vijayalakshmi R, Parameswari P. Ethnomedicinal survey of Jawadhu hills in Tamil Nadu. Asian J Pharm Clin Res 2012;2:45-9.

11. Johnsy G, Sargunam SD, Kaviyarasan V. Indigenous knowledge of medicinal plants used for the treatment of skin diseases by the Kaani tribe of Kanyakumari District. Int J Pharm Pharm Sci 2012;4:309-13.

12. Motlhanka DM, Nthoiwa GP. Ethnobotanical survey of medicinal plants of tswapong North, in Eastern Botswana: A case of plants from Mosweu and Seolwane villages. Eur J Med Plants 2013;3:10-24.

13. Banani D, Das TA, Dutta CN. A few traditional medicinal plants used as antifertility agents by ethnic people of Tripura, India. Int J Pharm Pharm Sci 2014;6:47-53.

14. Kankar SS, Ibrahim MH, Mustafa M, Rusea G. Ethnobotanical survey of medicinal plants used for traditional maternal healthcare in Katsina state, Nigeria. South Afr J Bot 2015;97:165-75.

15. Kodi P, Mwangi ME, Peter KC, Kariuki TS. Ethnobotanical survey of antimalarial medicinal plants used in Butebo County, Eastern Uganda. Eur J Med Plants 2017;21:1-22.

16. Aadhan K, Anand SP. Survey of medicinal plants used for the treatment of diabetes by the Paliyar's Tribe in Sadhuragiri hills, Tamil Nadu, India. Int J Herb Med 2017;5:17-25. 